08,11

\title{
Управление примесной микроструктурой, сформированной при неравновесном фазовом переходе в разбавленном трехкомпонентном сплаве
}

\author{
(C) А.А. Чеврычкина, А.Л. Корженевский
}

Институт проблем машиноведения РАН, Санкт-Петербург, Россия

E-mail: alekorzh@mail.ru

Поступила в Редакцию 6 июля 2020 г.

В окончательной редакции 6 июля 2020 г.

Принята к публикации 7 июля 2020 г.

На основе общего аналитического подхода, подкрепленного результатами модельных численных расчетов, в статье обсуждаются возможности создания периодических слоистых примесных структур, формирующихся при неравновесных фазовых переходах в разбавленных тройных сплавах, и целенаправленного управления их свойствами. Вычислены пространственные профили концентраций легирующих примесей в твердой фазепродукте.

Ключевые слова: многокомпонентные сплавы, фазовые переходы, градиент температуры, подвижные примеси, управление параметрами периодических примесных сверхструктур.

DOI: 10.21883/FTT.2020.12.50214.143

\section{1. Введение}

В настоящее время ведется интенсивный поиск новых методик для создания материалов, обладающих заданным поведением в отношении целого комплекса механических, физических и химических требований. Основные линии исследований соответствуют следующим направлениям: 1) варьирование составов (например, легирование сложных сталей), 2) управление микроструктурой (например, производство порошков металлических стекол или создание эвтектик) и 3) способы формообразования (например, 3D-печать или разнообразные варианты аддитивных технологий). Наша статья посвящена вопросам, относящимся к первому и второму направлениям для трехкомпонентных сплавов.

В случае сплавов требование удовлетворить сразу нескольким условиям на различные механические, физические и/или химические свойства с необходимостью приводит к использованию многокомпонентных составов. Среди них в силу технологических и экономических причин на первый план выходят разбавленные сплавы, в которых введение в небольшом количестве каждого легирующего элемента отвечает за появление того или иного требуемого качественного свойства. Эффективная методика производства таких сплавов подразумевает надежный контроль характеристик материала-продукта, а также максимально возможное сокращение времени технологического процесса для его создания.

Возможность варьирования большого количества параметров при формировании многокомпонентных сплавов потенциально перспективна, но она же сильно затрудняет определение совокупности конкретных процедур, обеспечивающих получение продукта с оптимальными характеристиками. В частности, непосредственное, проведенное без предварительного теоретического анализа, применение компьютерных расчетов к соответствующим многопараметрическим моделям представляется малоперспективным подходом.

В сплавах, испытывающих фазовые переходы (например, в сплавах на основе железа, титана и никеля), имеется интересная экспериментальная возможность создания регулярных микроструктур за счет использования подвижного градиента температуры, созданного специально или появляющегося, например, при остывании сплава. Подобные структуры давно были обнаружены в ряде бинарных металлических сплавов, образующихся при их быстрой направленной кристаллизации из расплавов [1-12]. Относительная простота состава таких сплавов позволила подтвердить численными методами факт появления таких структур при некоторых фиксированных значениях модельных параметров [9-12].

Сравнительно недавно условия возникновения и периодическую пространственную зависимость профилей концентрации от значений термодинамических и кинетических параметров разбавленных бинарных сплавов удалось приближенно рассчитать аналитически, причем было получено близкое соответствие аналитических результатов с результатами численных расчетов [13-22]. Это относится как к описанию регулярных слоистых примесных структур, формирующихся при быстрой направленной кристаллизации [13-21], так и к подобным структурам, возникающим при твердотельных фазовых переходах [22]. 
В настоящей статье на примере трехкомпонентного разбавленного сплава мы покажем, что обобщение предложенного в [13-22] метода позволяет путем выполнения весьма громоздких, но в принципе несложных расчетов найти аналитически параметры периодических слоистых примесных структур в многокомпонентных сплавах-продуктах в зависимости от термодинамических и кинетических свойств исходного сплава, а также заданной величины подвижного градиента температуры. В частности, мы определили в явном виде зависимости формирующихся профилей концентрации обеих легирующих компонент от их энергии взаимодействия, отношения коэффициентов диффузии и коэффициентов распределения примеси. Полученные приближенные аналитические зависимости подтверждены результатами численных расчетов.

\section{2. Формулировка модели и исходных уравнений движения межфазной границы и легирующих примесей}

В нашей статье [19] было аналитически показано, что для ряда разбавленных металлических сплавов в плоскости управляющих параметров градиент температуры скорость его перемещения $\left(\nabla T-v_{p}\right.$ существует большая область значений этих параметров, в которой движение плоской межфазной границы (МФГ) морфологически устойчиво, и следовательно, может быть описано в рамках одномерной модели. В качестве такой модели для тройного сплава, испытывающего фазовый переход с изменением концентраций, мы будем использовать следующее выражение для плотности свободной энергии

$$
H=\frac{\rho(T)}{2} \int d z\left\{\sum_{i=1}^{2}\left[C_{i}-C_{i, e q}(z-Z)\right]^{2}+A C_{1} C_{2}\right\},
$$

где $C_{i}$ - концентрации примесей, коэффициент $A$ отражает их взаимодействие, функции $C_{i, e q}(z-Z)$ описывают равновесные распределения примесей в присутствии границы при $A=0, Z$ - координата границы. Для разбавленных сплавов функция $\rho(T)$ имеет вид

$$
\rho(T)=-\left(\frac{\partial C}{\partial T}\right)^{-1} \frac{L}{T_{c}} \frac{1}{\Delta C(T)},
$$

где $L, T_{c}$ и $\Delta C(T)$ обозначают теплоту превращения, температуру перехода и ширину области концентрационного гистерезиса соответственно. Чтобы избежать излишней громоздкости дальнейших формул, мы не стали вводить в $(1),(2)$ функций $\rho_{i}(T)$. различных для обеих компонент примеси, что не сказывается на основных качественных результатах, полученных ниже.

Уравнения движения в модели (1), принадлежащей к семейству капиллярно-волновых моделей [23], запи- шем в виде

$$
\begin{aligned}
& \partial_{t} Z=\Lambda\left(F-\frac{\partial H}{\partial Z}\right), \\
& \partial_{t} C_{i}=\left(\frac{D_{i}}{\rho}\right) \nabla^{2} \frac{\delta H}{\delta C_{i}} .
\end{aligned}
$$

Здесь $\Lambda$ - кинетический коэффициент, описывающий движение МФГ, $D_{i}$ - коэффициенты диффузии. Опятьтаки ради упрощения записи формул мы ограничились описанием диффузии примесей классическим параболическим уравнением. Движущая сила $F$, действующая на границу, определяется как $F=L\left(T_{c}-T\right) / T_{c}$. Значение локальной температуры $T$ определяется заданной средней скоростью границы $v_{p}$ и фиксированным градиентом температуры $G$

$$
T=T_{c}+G\left(z-v_{p} t\right)
$$

Характерные пространственные масштабы изменения равновесных распределений $C_{i, e q}(z)$ мы будем считать одинаковыми по порядку величины и обозначать буквой $\xi$.

Для проведения промежуточных расчетов удобно ввести безразмерные переменные

$$
\frac{z}{\xi} \rightarrow z, \quad \frac{D_{1} t}{\xi^{2}} \rightarrow t, \quad \frac{2\left(C_{i}-C_{i 0}\right)}{\Delta C} \rightarrow C_{i}
$$

и независимые безразмерные параметры

$$
\begin{gathered}
\gamma_{1}=\left(\frac{\Delta C}{2}\right)^{2} \frac{\rho \xi}{\sigma}, \quad \gamma_{2}=d \gamma_{1}, \quad d=\frac{D_{2}}{D_{1}}, \\
p=\frac{v_{c}}{v_{d}}, \quad g=\left(\frac{\xi^{3} L}{k T_{c}}\right)\left(\frac{\xi G}{T_{c}}\right),
\end{gathered}
$$

где $k$ - постоянная Больцмана, $C_{i 0}-$ концентрации примесей в исходной фазе, $\sigma$ - коэффициент поверхностного натяжения, $v_{c}$ - максимальное значение скорости перемещения МФГ в чистом кристалле, $v_{d}=D_{1} / \xi-$ максимальная скорость диффузионных скачков атомов примеси $C_{1}$. Отметим, что обычно $v_{d}$ много меньше скорости $v_{c}$, т. е. параметр $p \gg 1$.

Во введенных переменных уравнения движения плоской границы приобретают вид

$$
\begin{gathered}
\frac{1}{\rho} \partial_{t} Z=F(Z)-\sum_{i=1}^{2} \gamma_{i} \int_{-\infty}^{+\infty} d z C_{i, e q}^{\prime}(z-Z(t)) \\
\times\left[C_{i}(z, t)-C_{i, e q}(z-Z(t))\right], \\
\partial_{t} C_{1}=D_{1} \partial_{z z}\left[C_{1}-C_{1, e q}(z-Z(t))+A C_{2}\right], \\
\partial_{t} C_{2}=D_{2} \partial_{z z}\left[C_{2}-C_{2, e q}(z-Z(t))+A C_{1}\right] .
\end{gathered}
$$




\section{3. Представление матричной функции Грина для диффузионных уравнений в виде разложения в бесконечный ряд по скорости МФГ и ее временным производным}

Поскольку нас интересуют регулярные пространственно-периодические структуры в фазе-продукте, которые появляются вследствие установившихся периодических колебаний скорости МФГ вокруг постоянного заданного значения скорости смещения изотерм $v_{p}$, то естественно выбрать решение диффузионных уравнений (8) с граничными условиями $C_{i}(z, t=-\infty)=0$. Выполнив свертку функции Грина $\mathscr{F}_{i j}$ с источниками $D_{i} \partial_{z}^{2} C_{i, e q}$ в уравнениях (8), запишем их решение в виде

$$
\begin{aligned}
& C_{i}(z, t)=D_{i} \int_{-\infty}^{t} d t^{\prime} \\
& \quad \times \int_{-\infty}^{+\infty} d z^{\prime} \partial_{z^{\prime}} \mathscr{F}_{i j}\left(z-z^{\prime}, t-t^{\prime}\right) \partial_{z^{\prime}} C_{i, e q}\left(z^{\prime}-Z\left(t^{\prime}\right)\right) .
\end{aligned}
$$

В этом решении удобно использовать представление функции Грина диффузионных уравнений (8) в виде

$$
\mathscr{F}_{i j}(z, t)=\int_{-\infty}^{+\infty} \frac{d q}{2 \pi} \mathscr{F}_{i j}(q, t) e^{i q z}
$$

где элементы матрицы $\mathscr{F}_{i j}(q, t)$ имеют вид

$$
\begin{aligned}
& \mathscr{F}_{11}(q, t) \\
& =\frac{\left(i \omega_{-}+D_{2} q^{2}\right) \exp \left(i \omega_{-} t\right)-\left(i \omega_{+}+D_{2} q^{2}\right) \exp \left(i \omega_{+} t\right)}{q^{2} \sqrt{\left(D_{1}-D_{2}\right)^{2}+4 A^{2} D_{1} D_{2}}}, \\
& =\frac{\left(i \omega_{-}+D_{1} q^{2}\right) \exp \left(i \omega_{-} t\right)-\left(i \omega_{+}+D_{1} q^{2}\right) \exp \left(i \omega_{+} t\right)}{q^{2} \sqrt{\left(D_{1}-D_{2}\right)^{2}+4 A^{2} D_{1} D_{2}}}, \\
& \mathscr{F}_{12}(q, t)=D_{2} A \frac{\exp \left(i \omega_{+} t\right)-\exp \left(i \omega_{-} t\right)}{\sqrt{\left(D_{1}-D_{2}\right)^{2}+4 A^{2} D_{1} D_{2}}}, \\
& \mathscr{F}_{21}(q, t)=D_{1} A \frac{\exp \left(i \omega_{+} t\right)-\exp \left(i \omega_{-} t\right)}{\sqrt{\left(D_{1}-D_{2}\right)^{2}+4 A^{2} D_{1} D_{2}}} .
\end{aligned}
$$

Ее запись можно упростить, если вместо выражений для частот $\omega_{+}$и $\omega_{-}$

$$
\omega_{ \pm}=\frac{i q^{2}}{2}\left(\left(D_{1}+D_{2}\right) \pm \sqrt{\left(D_{1}-D_{2}\right)^{2}+4 A^{2} D_{1} D_{2}}\right)
$$

использовать безразмерные частоты $\Omega_{+}$и $\Omega_{-}$

$$
i \omega_{ \pm} \equiv-D_{1} q^{2} \Omega_{ \pm}
$$

где

$$
2 \Omega_{ \pm} \equiv(1+d) \pm \sqrt{(1-d)^{2}+4 A^{2} d} .
$$

Введя еще один безразмерный параметр

$$
R \equiv \sqrt{(1-d)^{2}+4 A^{2} d}
$$

получаем соотношения

$$
\Omega_{+}-\Omega_{-}=R, \quad \Omega_{+}+\Omega_{-}=1+d .
$$

Это позволяет представить элементы матрицы $\mathscr{F}_{i j}(q, t)$ в компактной форме, облегчающей последующие вычисления:

$$
\begin{aligned}
\mathscr{F}_{11}(q, t) & =\frac{\left(-\Omega_{-}+d\right) \exp \left(i \omega_{-} t\right)-\left(-\Omega_{+}+d\right) \exp \left(i \omega_{+} t\right)}{R} \\
& \equiv \mathscr{F}_{11,-}-\mathscr{F}_{11,+},
\end{aligned}
$$

где

$$
\begin{gathered}
\mathscr{F}_{11,-} \equiv \frac{\left(-\Omega_{-}+d\right) \exp \left(i \omega_{-} t\right)}{R}, \\
\mathscr{F}_{11,+} \equiv \frac{\left(-\Omega_{+}+d\right) \exp \left(i \omega_{+} t\right)}{R},
\end{gathered}
$$

Аналогичным образом представляем остальные матричные элементы:

$$
\begin{aligned}
\mathscr{F}_{22}(q, t) & =\frac{\left(-\Omega_{-}+1\right) \exp \left(i \omega_{-} t\right)-\left(-\Omega_{+}+1\right) \exp \left(i \omega_{+} t\right)}{R} \\
& \equiv \mathscr{F}_{22,-}-\mathscr{F}_{22,+}, \\
\mathscr{F}_{12}(q, t) & =d A \frac{\exp \left(i \omega_{+} t\right)-\exp \left(i \omega_{-} t\right)}{R} \equiv \mathscr{F}_{12,-}-\mathscr{F}_{12,+}, \\
\mathscr{F}_{21}(q, t) & =A \frac{\exp \left(i \omega_{+} t\right)-\exp \left(i \omega_{-} t\right)}{R} \equiv \mathscr{F}_{21,-}-\mathscr{F}_{21,+} \cdot
\end{aligned}
$$

Введя координаты $\xi, \xi^{\prime}$, отсчитанные от положения МФГ в моменты времени $t, t^{\prime}$,

$$
\xi \equiv z-Z(t), \quad \xi^{\prime}=z^{\prime}-Z\left(t^{\prime}\right)
$$

и обозначив для краткости функцию $C_{i, e q}^{\prime}(\zeta) \equiv a_{i}(\xi)$ представим значения концентраций $C_{i}(z, t)$ в виде выражений, содержащих в явном виде зависимость от разности координат МФГ

$$
\begin{aligned}
C_{i}(z, t)= & \int_{0}^{\infty} d \tau \int_{-\infty}^{+\infty} d \xi^{\prime} \partial_{\xi^{\prime}} \mathscr{F}_{i j} \\
& \times\left(\xi-\xi^{\prime}+\left(Z(t)-Z\left(t^{\prime}\right)\right), \tau\right) D_{j} a_{j}\left(\xi^{\prime}\right),
\end{aligned}
$$

где $\tau=t-t^{\prime}$. Используя в формуле (18) для функции Грина ее представление (10), разложим в показателе экспоненты разность $Z(t)-Z\left(t^{\prime}\right)$ по степеням $\tau$ :

$$
Z(t)-Z\left(t^{\prime}\right)=\dot{Z}(t) \tau-\frac{1}{2} \ddot{Z}(t) \tau^{2}+\frac{1}{6} \dddot{Z}(t) \tau^{3}+\cdots
$$

Из (18), (19) становится очевидным, в чем состоит преимущество работы с представлением (10): несмотря 
на то, что вид траектории $Z(t)$ пока никак не определен, мы, тем не менее, можем выполнить в (18) интегрирование по времени в явном виде, так как при этой операции функции $\dot{Z}(t), \ddot{Z}(t), \ldots$, играют роль пассивных параметров.

Продемонстрируем, как это конкретно выглядит на примере матричного элемента $\mathscr{F}_{11,-}$ :

$$
\begin{aligned}
& \mathscr{F}_{11,-}\left(z-z^{\prime}, t-t^{\prime}\right)=\frac{\left(-\Omega_{-}+d\right)}{R} \int_{-\infty}^{+\infty} \frac{d q}{2 \pi} \\
& \quad \times \exp \left[-q^{2} D_{1} \Omega_{-}\left(t-t^{\prime}\right)+i q\left(\xi-\xi^{\prime}\right)+i q \dot{Z}(t)\left(t-t^{\prime}\right)\right] \\
& \quad \times \exp \left\{-i q\left[\frac{1}{2} \ddot{Z}(t)\left(t-t^{\prime}\right)^{2}+\frac{1}{6} \dddot{Z}(t)\left(t-t^{\prime}\right)^{3}+\cdots\right]\right\} .
\end{aligned}
$$

В соответствии с нашим общим подходом к аналитическому описанию предполагаемой динамики МФГ в виде релаксационных колебаний, сформулированному в $[20,22]$, допустим, что вклад в общее смещение границы $Z(t)$ от движения на участках с большим ускорением мал из-за малой длительности таких участков относительно полного периода колебаний. Поэтому в показателе последней экспоненты в (20) оставим только член $\sim \ddot{Z}(t)\left(t-t^{\prime}\right)^{2}$, после чего разложим ее, ограничиваясь лишь двумя первыми членами

$$
\exp \left[-i q \frac{1}{2} \ddot{Z}(t)\left(t-t^{\prime}\right)^{2}\right] \approx 1-i q \frac{1}{2} \ddot{Z}(t)\left(t-t^{\prime}\right)^{2} .
$$

Интеграл по времени $\tau$ для вклада $\mathscr{F}_{11,-}$ в (9) запишем в виде

$$
\begin{gathered}
\int_{0}^{+\infty} d \tau \exp \left[\left(-q^{2} D_{1} \Omega_{-}+i q \dot{Z}(t)\right) \tau\right]\left\{1-i q \frac{1}{2} \ddot{Z}(t) \tau^{2}\right\} \\
\equiv J_{0}^{1-}+J_{2}^{1-}
\end{gathered}
$$

где

$$
\begin{gathered}
J_{0}^{1-}=\int_{0}^{+\infty} d \tau \exp \left[\left(-q^{2} D_{1} \Omega_{-}+i q \dot{Z}(t)\right) \tau\right] \\
=\frac{1}{\left(q^{2} D_{1} \Omega_{-}-i q v\right)}, \\
J_{2}^{1-}=-i q \frac{1}{2} \ddot{Z}(t) \int_{0}^{+\infty} d \tau \exp \left[\left(-q^{2} D_{1} \Omega_{-}+i q \dot{Z}(t)\right) \tau\right] \tau^{2} \\
=\frac{i \ddot{Z}(t)}{2 q} \frac{\partial^{2}}{\partial v^{2}} \int_{0}^{\infty} d \tau \exp \left[\left(-q^{2} D_{1} \Omega_{-}+i q v\right) \tau\right] \\
=\frac{i \ddot{Z}(t)}{2 q} \frac{\partial^{2}}{\partial v^{2}} \frac{1}{\left(q^{2} D_{1} \Omega_{-}-i q v\right)}=\frac{i \ddot{Z}(t) \partial}{2 q} \frac{\partial^{2}}{\partial v^{2}} J_{0}^{1-} .
\end{gathered}
$$

Здесь $v=d Z / d t$. Отметим, что последнее соотношение между интегральными вкладами, зависящими только от скорости МФГ, и вкладами, пропорциональными ускорению, выполняется для всех матричных элементов

$$
J_{2}^{i, \pm}=i \frac{\ddot{Z}}{2 q} \frac{\partial^{2} J_{0}^{i, \pm}}{\partial v^{2}} .
$$

Проинтегрировав теперь зависящую только от скорости часть вклада в $\mathscr{F}_{11,-}\left(z-z^{\prime}, t-t^{\prime}\right)$ по $q$, получим

$$
\begin{aligned}
& \int_{-\infty}^{+\infty} \frac{d q}{2 \pi} \frac{-i}{\left(q D_{1} \Omega_{-}-i v\right)} \exp \left[i q\left(\xi-\xi^{\prime}\right)\right] \\
& =\frac{\Theta\left(\xi-\xi^{\prime}\right)}{D_{1} \Omega_{-}} \exp \left[-\frac{v}{D_{1} \Omega_{-}}\left(\zeta-\xi^{\prime}\right)\right],
\end{aligned}
$$

где $\Theta\left(\xi-\xi^{\prime}\right)-$ функция Хэвисайда. Используя связь (25), для пропорциональной ускорению $\ddot{Z}$ части вклада, в свою очередь, получаем

$$
\begin{aligned}
& \frac{\ddot{Z}}{2} \frac{\partial^{2}}{\partial v^{2}} \int_{-\infty}^{+\infty} \frac{d q}{2 \pi} \frac{\exp \left[i q\left(\xi-\xi^{\prime}\right)\right\}}{(q+i \epsilon)\left(q D_{1} \Omega_{+}-i v\right)} \\
& =\frac{\ddot{Z}}{2} \frac{\partial^{2}}{\partial v^{2}} \frac{1}{v}\left(\Theta\left(\xi-\zeta^{\prime}\right) \exp \left[-\frac{v}{D_{1} \Omega_{-}}\left(\xi-\zeta^{\prime}\right)\right]+\Theta\left(\xi^{\prime}-\zeta\right)\right) .
\end{aligned}
$$

Аналогичным образом мы вычислили и все остальные подобные вклады в функцию Грина. В принципе, так можно вычислить коэффициенты перед ускорениями сколь угодно высоких порядков в точном разложении этой функции в ряд по производным скорости. Однако, как уже упоминалось выше, поскольку мы интересуемся динамикой границы, предположительно движущейся в режиме релаксационных колебаний, то в ряду для функции Грина в (18) можно сохранить лишь члены, зависящие от скорости $v$ и ускорения $\dot{v}$, т.к. относительные вклады в полное смещение границы $Z(t)$ от членов, пропорциональных ускорениям более высоких порядков $\ddot{v}, \dddot{v}, \ldots$, для колебаний такого типа малы.

\section{4. Общее выражение для динамических профилей концентрации легирующих примесей и его модельный вид}

Как следует непосредственно уже из самой формы записи свертки (18), выведенное в предыдущем параграфе разложение функции Грина в ряд по производным скорости МФГ автоматически означает, что аналогичные разложения возникают в этом представлении и для пространственно-временных профилей концентраций легирующих примесей $C_{i}(z, t)$ Подставляя в $(18)$ приведенное выше приближенное выражение для $\mathscr{F}_{i j}$ и 
выполняя интегрирование по координате $\xi^{\prime}$ получаем, что эти профили могут быть записаны в общем виде

$$
\begin{aligned}
& C_{1}(\xi, t) \equiv C_{1}^{(1)}+C_{1}^{(2)}, \quad C_{2}(\xi, t) \equiv C_{2}^{(1)}+C_{2}^{(2)}, \\
& C_{1}^{(1)}=C_{11}^{+}(\xi ; v)+C_{11}^{-}(\xi ; v)+C_{12}^{+}(\xi ; v)+C_{12}^{-}(\xi ; v), \\
& C_{1}^{(2)}=\frac{\dot{v}}{2} \frac{\partial^{2}}{\partial v^{2}}\left\{\frac { D _ { 1 } } { v } \left[\left(\Omega_{+}\left(C_{11}^{+}(\xi ; v)+C_{11}^{+}(-\xi ; 0)\right)\right.\right.\right. \\
&+\Omega_{-}\left(C_{11}^{-}(\xi ; v)+C_{11}^{-}(-\xi ; 0)\right)+\Omega_{+}\left(C_{12}^{+}(\xi ; v)\right. \\
&\left.\left.\left.+C_{12}^{+}(-\xi ; 0)\right)+\Omega_{-}\left(C_{12}^{-}(\xi ; v)+C_{12}^{-}(-\xi ; 0)\right)\right]\right\}, \\
& C_{2}^{(1)}(\xi, t)=C_{22}^{+}(\xi ; v)+C_{22}^{-}(\xi ; v)+C_{21}^{+}(\xi ; v)+C_{21}^{-}(\xi ; v), \\
& C_{2}^{(2)}= \frac{\dot{v}}{2} \frac{\partial^{2}}{\partial v^{2}}\left\{\frac { D _ { 1 } } { v } \left[\Omega_{+}\left(C_{22}^{+}(\xi ; v)+C_{22}^{+}(-\xi ; 0)\right)\right.\right. \\
&+\Omega_{-}\left(C_{22}^{-}(\xi ; v)+C_{22}^{-}(-\xi ; 0)\right) \\
&+\Omega_{+}\left(C_{21}^{+}(\xi ; v)+C_{21}^{+}(-\xi ; 0)\right) \\
&\left.+\Omega_{-}\left(C_{21}^{-}(\xi, v)+C_{21}^{-}(-\xi ; 0)\right)\right],
\end{aligned}
$$

где вклады $C_{i j}^{ \pm}(\xi ; v)$ определены как

$$
C_{i j}^{ \pm}(\xi ; v) \equiv \int_{0}^{\infty} d \tau \int_{-\infty}^{+\infty} d \xi^{\prime} \partial_{\xi^{\prime}} \mathscr{F}_{i j}^{ \pm}\left(\xi-\xi^{\prime}+v \tau\right)\left(D_{j} a_{j}\left(\xi^{\prime}\right)\right) \text {, }
$$

причем суммирование по повторяющимся индексам в этой формуле не подразумевается. Отметим, что вид формулы (29) формально совпадает с выражениями для вкладов в стационарные профили, когда движение МФГ происходит с постоянной скоростью. Важно, однако, помнить, что в формулах (28) временная зависимость $v(t)$ пока еще не была определена.

Очевидная громоздкость этих формул не должна удивлять, т.к. они описывают динамические профили концентраций взаимодействующих легирующих примесей в тройном сплаве, причем коэффициенты диффузии $D_{i}$ и равновесные профили концентраций $C_{i, e q}(z)$ предполагаются различными.

В дальнейшем при проведении численных расчетов мы используем простую модель, в которой функции $a_{i}(\xi)$ выбраны в виде

$$
a_{i}(\xi)=a_{i} \Theta(\epsilon-\xi) \Theta(\epsilon+\zeta) .
$$

Этот выбор отвечает линейному изменению равновесных профилей концентраций в пределах границы, имеющей ширину $\xi=2 \epsilon$ и он позволяет рассчитать все вклады, входящие в (28), аналитически. Ввиду большой громоздкости выражений для модельных вкладов $C_{i}^{(2)}$ мы приводим в этом разделе в явном виде лишь простые формулы для размерных вкладов $C_{i}^{(1)}$, а громоздкие выражения для вкладов $C_{i}^{(2)}$ поместили в Приложение:

$$
\begin{aligned}
& C_{1}^{(1)}(\xi ; v)=\frac{a_{1} D_{1}}{v} \\
& +\frac{1}{R v}\left(a_{1} D_{1}\left(-\Omega_{+}+d\right)-a_{2} D_{2} d A\right) \exp \left[-\frac{v}{D_{1} \Omega_{+}}(\xi+\epsilon)\right] \\
& +\frac{1}{R v}\left(-a_{1} D_{1}\left(-\Omega_{+}+d\right)+a_{2} D_{2} d A\right) \exp \left[-\frac{v}{D_{1} \Omega_{-}}(\xi+\epsilon)\right], \\
& C_{2}^{(1)}(\xi ; v)=\frac{a_{2} D_{2}}{v} \\
& +\frac{1}{R v}\left(a_{2} D_{2}\left(-\Omega_{+}+1\right)-a_{1} D_{1} A\right) \exp \left[-\frac{v}{D_{1} \Omega_{+}}(\xi+\epsilon)\right] \\
& +\frac{1}{R v}\left(-a_{2} D_{2}\left(-\Omega_{-}+1\right)+a_{1} D_{1} A\right) \exp \left[-\frac{v}{D_{1} \Omega_{-}}(\xi+\epsilon)\right] .
\end{aligned}
$$

После подстановки профилей (28) в уравнение движения МФГ (7), принимающее при этом вид уравнения для нелинейного осциллятора, также в явной аналитической форме удается вычислить эффективные массу и силу трения. В свою очередь, подставив решения этого уравнения $Z(t)$ в формулы (28), мы получим выражения для профилей $C_{i}(z, t)$ в явном виде. Вопросам динамики МФГ и индуцированным ею пространственным распределениям примесей посвящен следующий раздел.

\section{5. Уравнение движения межфазной границы, его автоколебательные решения и расчет пространственно-периодических примесных профилей}

Выполнив интегрирование по координате $\xi$ в уравнении движения МФГ (7) для профилей $(28-30)$, мы получаем его представление в виде уравнения для нелинейного осциллятора, обладающего нелинейно зависящими от скорости силой трения и массой

$$
M(v) \ddot{Z}+r(v)+g\left(Z-v_{p} t\right)-F=0,
$$

где играющая роль вязкого нелинейного трения сила $r(v)$ равна

$$
\begin{array}{r}
r(v) \equiv \frac{v}{p}-f_{1}(v)-f_{2}(v)+f_{1}(0)+f_{2}(0), \\
f_{i}(v) \equiv-\gamma_{i} \int_{-\infty}^{+\infty} d \xi C_{i, e q}^{\prime}(\xi) C_{i}^{(1)}(\xi, v) .
\end{array}
$$


В явном виде модельные выражения для $f_{i}(v)$ записываются так:

$$
\begin{aligned}
& f_{1}(v)=-\gamma_{1} a_{1}\left[\frac{2 \epsilon a_{1} D_{1}}{v}-\frac{D_{1} \Omega_{+}}{R v^{2}}\right. \\
& \times\left(a_{1} D_{1}\left(-\Omega_{+}+d\right)-a_{2} D_{2} d A\right)\left(\exp \left[-\frac{2 \epsilon v}{D_{1} \Omega_{+}}\right]-1\right) \\
& -\frac{D_{1} \Omega_{-}}{R v^{2}}\left(a_{1} D_{1}\left(-\Omega_{-}+d\right)+a_{2} D_{2} d A\right)\left(\exp \left[-\frac{2 \epsilon v}{D_{1} \Omega_{-}}\right]-1\right) \\
& f_{2}(v)=-\gamma_{2} a_{2}\left\{\frac{2 \epsilon a_{2} D_{2}}{v}-\frac{D_{1} \Omega_{+}}{R v^{2}}\left(a_{2} D_{2}\left(-\Omega_{+}+1\right)\right.\right. \\
& \left.-a_{1} D_{1} A\right)\left(\exp \left[-\frac{2 \epsilon v}{D_{1} \Omega_{+}}\right]-1\right)-\frac{D_{1} \Omega_{-}}{R v^{2}} \\
& \left.\times\left(-a_{2} D_{2}^{\prime}\left(-\Omega_{-}+1\right)+a_{1} D_{1} A\right)\left(\exp \left[-\frac{2 \epsilon v}{D_{1} \Omega_{-}}\right]-1\right)\right\}
\end{aligned}
$$

Эффективная масса $M(v)$ в (32) вычисляется интегрированием по координате $\xi$ обоих „инерционных“ вкладов $C_{i}^{(2)}(\xi ; v)$ с их последующим сложением. В явном виде, однако, результирующее выражение слишком громоздко, поэтому оно также вынесено в Приложение. Мы использовали это выражение в последующих численных решениях уравнения $(32)^{1}$.

Учитывая, что при постоянной скорости $Z=v_{p} t$ выполняется условие баланса силы трения и движущей силы $r\left(v_{p}\right)=F$, удобно записать уравнение (32) для отклонения $h(t)=Z(t)-v_{p} t$ в виде обобщенного уравнения Рэлея

$$
M\left(v_{p}+\dot{h}\right) \ddot{h}+R\left(v_{p}+\dot{h}\right)+g h=0,
$$

где $R(v) \equiv r(v)-r\left(v_{p}\right)$. Фиксированная точка $h=\dot{h}=0$ становится неустойчивой, если сила трения обладает падающей ветвью, т.е. $R^{\prime}<0$. В этом случае притягивающим аттрактором решений уравнения (36) является устойчивый цикл, и в установившемся режиме динамика отклонений МФГ от подвижной изотермы является автоколебательной. Подстановка такого решения в выражения для концентраций $C_{i}(\xi, t)(28)$ показывает, что на границе (при $\xi=0$ ) их значения также испытывают автоколебания. Поскольку в реальных сплавах коэффициент диффузии примесей в твердой фазе мал, то с очень хорошей точностью можно считать, что значения $C_{i}\left(z=\int v(t) d t\right)$ описывают пространственные распределения легированных примесей в материалепродукте.

Использование самого уравнения движения МФГ в виде (32) (или (36)) оказывается также очень хорошим приближением, т. к. экспериментальные значения реальных температурных градиентов, записанные в безразмерных единицах, много меньше единицы как в случае

\footnotetext{
${ }^{1}$ В формуле (20) статьи [20] для эффективной массы бинарного сплава есть опечатка: в скобках знак должен быть плюс.
}

Значения максимальных скоростей на границе области автоколебательной динамики МФГ и периодов колебаний для разных наборов материальных параметров сплавов

\begin{tabular}{c|c|c|c|c|c}
\hline$A$ & $d$ & $a_{2} / a_{1}$ & $R^{\prime}=0$ & $v_{p}$ & $T\left(v_{p}\right)$ \\
\hline 0 & 10 & 2 & 2.19 & 0.05 & 3848902 \\
\hline 0.9 & 1 & 2 & 0.86 & $\begin{array}{r}0.6 \\
0.4\end{array}$ & $\begin{array}{r}9051 \\
12660\end{array}$ \\
\hline 0.9 & 5 & 2 & 3.22 & $\begin{array}{l}0.6 \\
2.5\end{array}$ & $\begin{array}{r}71998 \\
17708\end{array}$ \\
\hline-0.9 & 5 & 2 & 5.87 & 2.5 & 61574 \\
\hline 0.9 & 5 & -2 & 5.87 & 2.5 & 67243
\end{tabular}

направленной кристаллизации из расплава, так и при твердотельных фазовых переходах. Более подробное обсуждение этого вопроса и численные оценки приведены в наших работах [20,22].

Положение на плоскости управляющих параметров $v-\nabla T$, а также форма устойчивого предельного цикла, зависят от величин трех безразмерных параметров: $A, d, a_{1} / a_{2}$, определяющих относительную энергию взаимодействия, отношение коэффициентов диффузии и равновесных коэффициентов распределения примесей, соответственно. Поскольку характеристики цикла, в свою очередь, задают период и пространственные профили примесей, то мы выполнили численные расчеты, наглядно демонстрирующие влияние параметров $A$, $d, a_{1} / a_{2}$ на распределение примесей в материалепродукте. Значения параметров, для которых были проведены некоторые из наших расчетов, приведены в таблице.

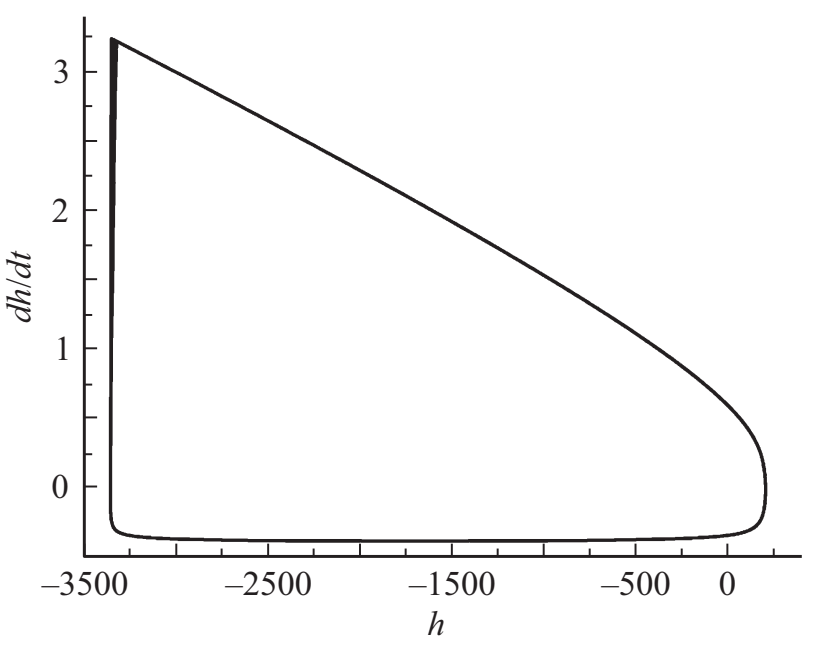

Рис. 1. Форма устойчивого цикла релаксационных колебаний МФГ при значениях параметров $d=1, a_{2} / a_{1}=2, a=0.9$, $v_{p}=0.4, g=6 \cdot 10^{-6}$. 
В таблице столбец $R^{\prime}=0$ с хорошей точностью определяет величину максимальной заданной средней скорости $v_{p}$, при которой движение МФГ имеет автоколебательный характер, в столбце $T\left(v_{p}\right)$ приведены значения периодов колебаний.

Типичная форма устойчивого цикла релаксационных колебаний МФГ приведена на рис. 1.

Близкая к плоской форма нижней границы цикла обеспечивает легкость выбора параметров режима, при работе в котором не возникает перекрытия колебательной моды с неустойчивостью Маллинза и Секерки и плоская МФГ остается морфологически устойчивой, что гарантирует формирование регулярной периодической слоистой структуры в сплаве-продукте.

Характер изменений пространственных профилей концентраций $C_{i}(z)$ при: а) значениях параметра энергии
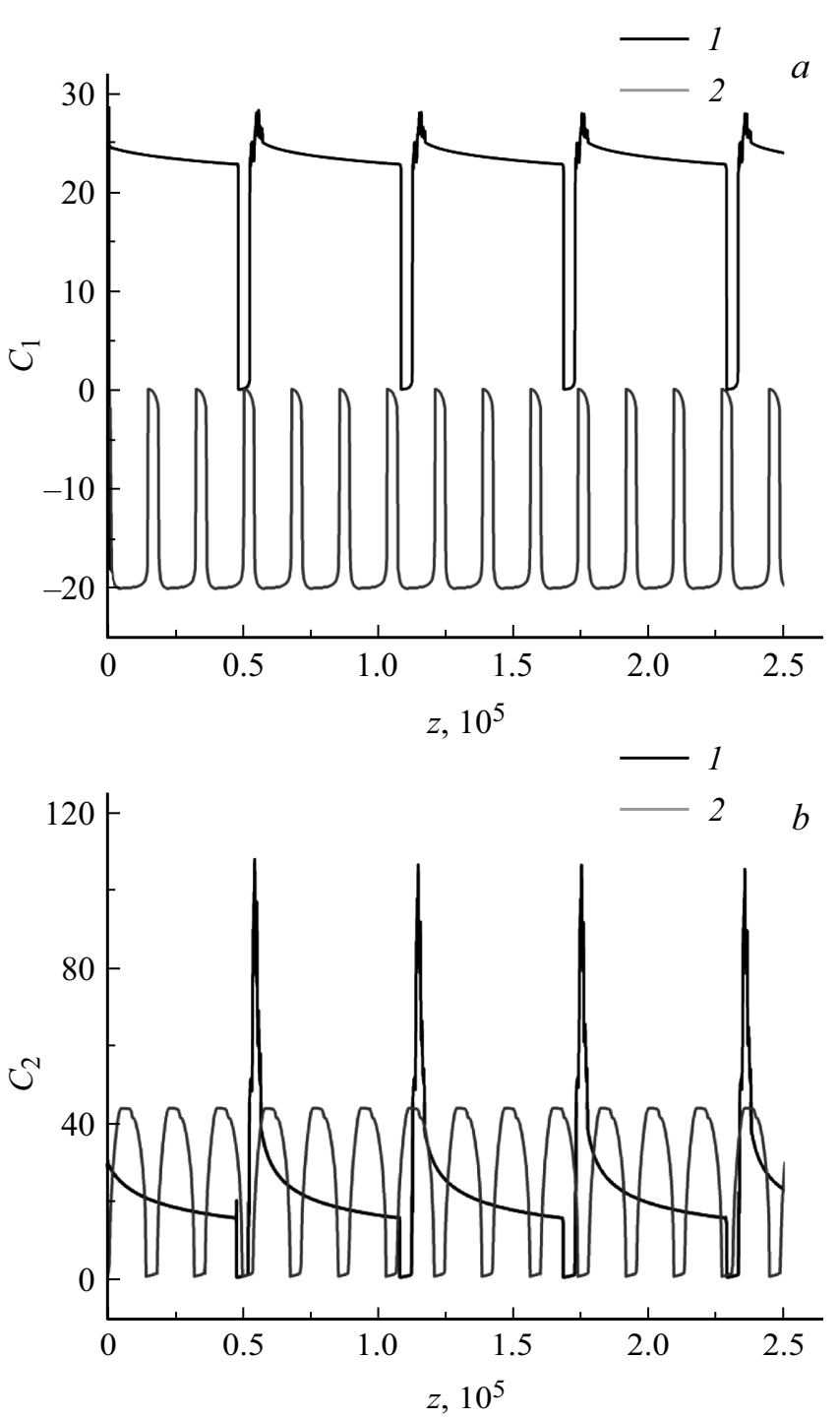

Рис. 2. Пространственные профили распределения легирующих примесей в сплаве-продукте при значениях параметров $\left.\left.d=5, a_{2} / a_{1}=2, v_{p}=2.5, g=6 \cdot 10^{-6}: a\right) C_{1}, b\right) c_{2}$. Линии 1 соответствует значение параметра $A=-0.9$, Линии $2-$ $A=0.9$.
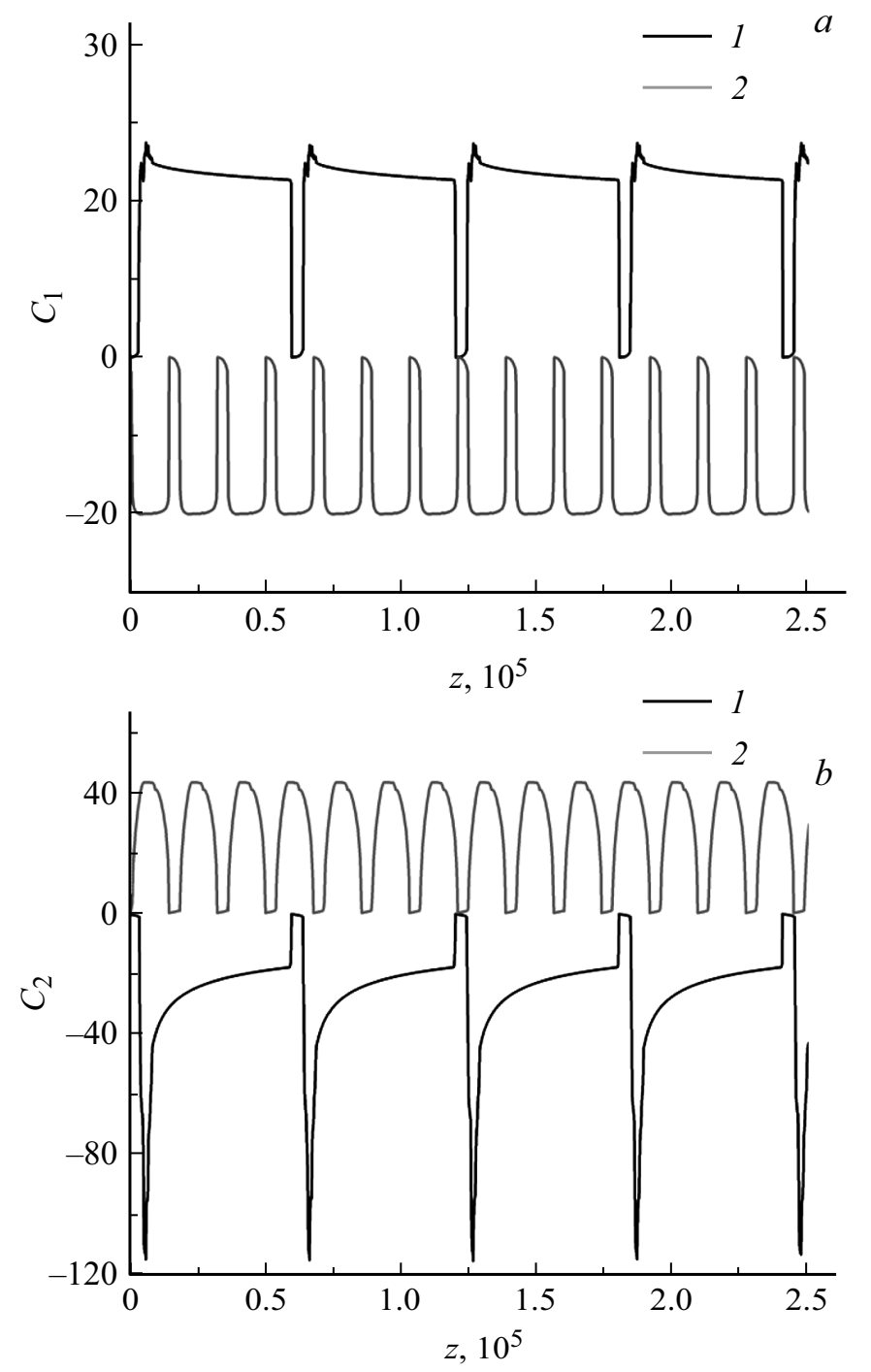

Рис. 3. Пространственные профили распределения легирующих примесей в сплаве-продукте при значениях параметров $\left.\left.d=5, A=0.9, v_{p}=2.5, g=6 \cdot 10^{-6}: a\right) C_{1}, b\right) c_{2}$. Линии 1 соответствует значение параметра $a_{2} / a_{1}=-2$, линии $2-$ $a_{2} / a_{1}=2$.

взаимодействия $A$, отвечающих случаям отталкивания и притяжения примесей, б) положительных и отрицательных значениях равновесных коэффициентов распределения примесей и в) разных значениях коэффициентов диффузии примесей проиллюстрирован на рис. 2-4.

Возможности более гибкого управления формой профилей при варьировании не одного, как на рис. 2-4, а, например, двух параметров (отношение коэффициентов диффузии и скорость $v_{p}$ ) проиллюстрированы на рис. 5 .

В частности, из него видно, что в случае отталкивающихся примесей $(A>0)$ максимумам концентрации примеси $C_{1}$ отвечают минимумы концентрации $C_{2}$, т. е. в динамическом режиме сохраняется тенденция к понижению энергии формирующейся структуры сплава. В целом, рис. 2-5 демонстрируют высокую чувстви- 

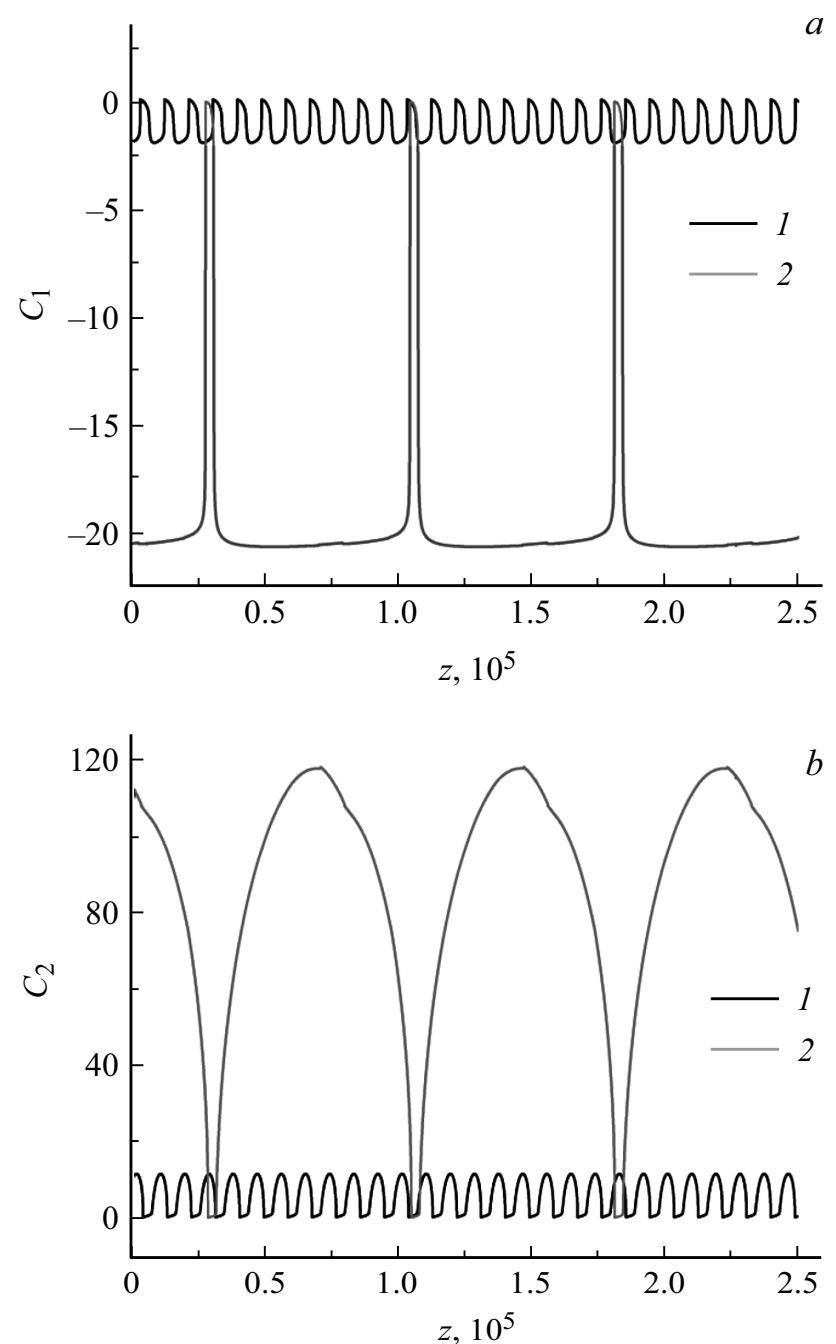

Рис. 4. Пространственные профили распределения легирующих примесей в сплаве-продукте при значениях параметров $\left.\left.a_{2} / a_{1}=2, A=0.9, v_{p}=0.6, g=6 \cdot 10^{-6}: a\right) C_{1}, b\right) c_{2}$. Линии 1 соответствует значение параметра $d=1$, линии $2-d=5$.

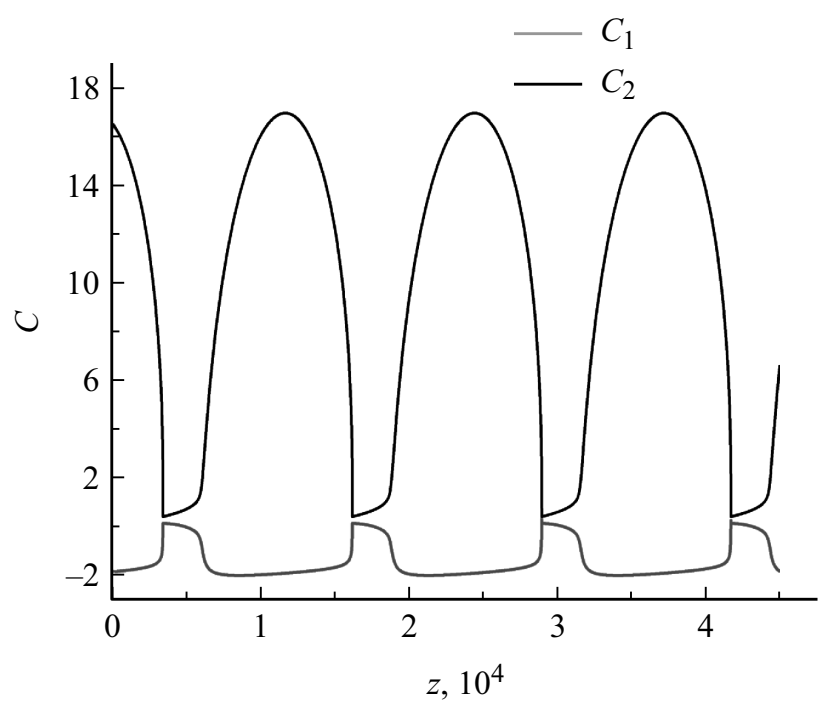

Рис. 5. Пространственные профили распределения легирующих примесей в сплаве-продукте при значениях параметров $d=1, a_{2} / a_{1}=2, A=0.9, v_{p}=0.4, g=6 \cdot 10^{-6}$. тельность формы примесных профилей к выбору пары легирующих примесей, обладающей фиксированным набором материальных констант.

\section{6. Заключение}

Применяя аналитические и численные методы, мы показали, что возможно целенаправленное регулирование формы периодических пространственных профилей легирующих примесей в разбавленных тройных сплавах, формирующихся в условиях неравновесных фазовых переходов. Это позволяет реализовать оптимальное управление всем комплексом требуемых свойств микроструктур в создаваемых сплавах-продуктах. Отметим, что описанная в работе совместная автоколебательная динамика межфазной границы и увлекаемых ею примесей обладает известной автономностью от внешних возмущений, что обеспечивает надежную воспроизводимость микроструктурных характеристик при изготовлении серийных изделий. Полученные результаты составляют общую аналитическую базу, которую целесообразно использовать при разработке эффективных технологий для создания новых микроструктурных материалов с заданным набором свойств.

\section{Финансирование работы}

Работа выполнена при финансовой поддержке Российского научного фонда (проект № 19-19-00552).

\section{Конфликт интересов}

Авторы заявляют, что у них нет конфликта интересов.

\section{Приложение}

Выражение для эффективной массы $M(v)$ в формуле (36) имеет следующий вид:

$$
M(v)=M_{1}(v)+M_{2}(v)+m(v),
$$

где

$$
\begin{gathered}
m(v)=\frac{2\left(D_{1} \gamma_{1} a_{1}^{2}+D_{2} \gamma_{2} a_{2}^{2}\right) \epsilon^{2}}{v^{3}}, \\
M_{1}(v)=\left(M_{11}^{+}+M_{11}^{-}\right)(v)+\left(M_{12}^{+}+M_{12}^{-}\right)(v), \\
M_{2}(v)=\left(M_{21}^{+}+M_{11}^{-}\right)(v)+\left(M_{22}^{+}+M_{12}^{-}\right)(v), \\
M_{11}^{ \pm}=\gamma_{1} a_{1}^{2} \Omega_{ \pm} \frac{\left(-\Omega_{ \pm}+d\right) D_{1}^{2}}{2 R} \frac{\partial^{2}}{\partial v^{2}} \\
\times\left\{\frac{2 \epsilon}{v^{2}}+\frac{D_{1} \Omega_{ \pm}}{v^{3}}\left[\exp \left(-\frac{2 \epsilon v}{D_{1} \Omega_{ \pm}}\right)-1\right]\right\}, \\
M_{12}^{ \pm}=-\gamma_{1} a_{1} a_{2} \Omega_{ \pm} \frac{A D_{2}^{2}}{2 R} \frac{\partial^{2}}{\partial v^{2}} \\
\times\left\{\frac{2 \epsilon}{v^{2}}+\frac{D_{1} \Omega_{ \pm}}{v^{3}}\left[\exp \left(-\frac{2 \epsilon v}{D_{1} \Omega_{ \pm}}\right)-1\right]\right\},
\end{gathered}
$$




$$
\begin{aligned}
M_{22}^{ \pm}= & \gamma_{2} a_{2}^{2} \Omega_{ \pm} \frac{\left(-\Omega_{ \pm}+1\right) D_{1}^{2}}{2 R} \frac{\partial^{2}}{\partial v^{2}} \\
& \times\left\{\frac{2 \epsilon}{v^{2}}+\frac{D_{1} \Omega_{ \pm}}{v^{3}}\left[\exp \left(-\frac{2 \epsilon v}{D_{1} \Omega_{ \pm}}\right)-1\right]\right\}, \\
M_{21}^{ \pm}= & -\gamma_{2} a_{1} a_{2} \Omega_{ \pm} \frac{A D_{1}^{2}}{2 R} \frac{\partial^{2}}{\partial v^{2}} \\
& \times\left\{\frac{2 \epsilon}{v^{2}}+\frac{D_{1} \Omega_{ \pm}}{v}\left[\exp \left(-\frac{2 \epsilon v}{D_{1} \Omega_{ \pm}}\right)-1\right]\right\} .
\end{aligned}
$$

Выражения для слагаемых $C_{i j}^{ \pm}(\xi ; v)$ в формуле $(28)$ приведены ниже

$$
\begin{gathered}
C_{11}^{ \pm}(\xi, v)=-a_{1} \frac{\left(-\Omega_{ \pm}+d\right) D_{1}}{R v}\left(1-\exp \left[-\frac{v}{D_{1} \Omega_{ \pm}}(\xi+\epsilon)\right]\right) \\
C_{12}^{ \pm}(\xi, v)=a_{2} \frac{d A D_{2}}{R v}\left(1-\exp \left[-\frac{v}{D_{1} \Omega_{ \pm}}(\xi+\epsilon)\right]\right) \\
C_{22}^{ \pm}(\xi, v)=-a_{2} \frac{\left(-\Omega_{ \pm}+1\right) D_{12}}{R v} \\
\times\left(1-\exp \left[-\frac{v}{D_{1} \Omega_{ \pm}}(\xi+\epsilon)\right]\right) \\
C_{21}^{ \pm}(\xi, v)=-a_{1} \frac{A D_{1}}{R v}\left(1-\exp \left[-\frac{v}{D_{1} \Omega_{ \pm}}(\xi+\epsilon)\right]\right) \\
C_{11}^{ \pm}(-\zeta, 0)=-a_{1} \frac{\left(-\Omega_{+}+d\right) D_{1}}{R \Omega_{ \pm}}(\epsilon-\xi) \\
C_{12}^{ \pm}(-\zeta ; 0)=a_{2} \frac{d^{2} A}{r \Omega_{ \pm}}(\epsilon-\zeta) \\
C_{22}^{ \pm}(-\zeta ; 0)=-a_{2} \frac{\left(-\Omega_{+}+1\right)}{R \Omega_{ \pm}} d(\epsilon-\zeta) \\
C_{21}^{ \pm}(-\zeta ; 0)=a_{1} \frac{A}{R \Omega_{ \pm}}(\epsilon-\xi)
\end{gathered}
$$

\section{Список литературы}

[1] W.J. Boettinger, D. Schechtman, R.J. Schaffer, F.S. Biancaniello. Met. Mater. Trans. A 15, 55 (1984).

[2] M. Gremaud, M. Carrard, W. Kurz. Acta Met. Mater. 38, 2587 (1990).

[3] M. Zimmermann, M. Carrard, M. Gremaud, W. Kurz. Mater. Sci. Eng. A 134, 1278 (1991).

[4] M. Gremaud, M. Carrard, W. Kurz. Acta Met. Mater. 39, 1431 (1991).

[5] M. Carrard, M. Gremaud, M. Zimmerman, W. Kurz. Acta Met. 40, 983 (1992).

[6] W. Kurz, R. Trivedi. Met. Mater. Trans. A 27, 625 (1996).

[7] A. Karma, A. Sarkissian. Phys. Rev. Lett. 27, 2616 (1992).

[8] A. Karma, A. Sarkissian. Phys. Rev. E 47, 513 (1993).

[9] K. Brattkus, D.I. Meiron. SIAM J. Appl. Math. 52, 1303 (1992).

[10] G.J. Merchant, R.J. Braun, K. Brattkus, S.H. Davis. SIAM J. Appl. Math. 52, 1279 (1992).

[11] M. Conti. Phys. Rev. E 56, R6267 (1997).

[12] M. Conti. Phys. Rev. E 58, 2071 (1998).
[13] A.L. Korzhenevskii, R. Bausch, R. Schmitz. Phys. Rev. Lett. 108, 046101 (2012).

[14] A.L. Korzhenevskii, R. Bausch, R. Schmitz. Phys. Rev. E 85, 021605 (2012).

[15] A.L. Korzhenevskii, R.E. Rozas, J. Horbach. J. Phys. 28, 035001 (2016).

[16] E. Babushkina, N.M. Bessonov, A.L. Korzhenevskii, R. Bausch, R. Schmitz. Phys. Rev. E 87, 042402 (2013).

[17] R.E. Rozas, A.L. Korzhenevskii, R. Bausch, R. Schmitz. Physica A 413, 394 (2014).

[18] R.E. Rozas, A.L. Korzhenevskii, J. Horbach. J. Phys. 28, 035001 (2016).

[19] А.А. Чеврычкина, Н.М. Бессонов, А.Л. Корженевский. ФTT 61, 1904 (2019).

[20] А.А. Чеврычкина, Н.М. Бессонов, А.Л. Корженевский. ФTT 61, 2122 (2019).

[21] A.A. Chevrychkina, N.M. Bessonov, A.L. Korzhenevskii, D.V. Alexandrov. Eur. Phys. J. Spec. Top. 229, 253 (2020).

[22] А.А. Чеврычкина, Н.М. Бессонов, А.Л. Корженевский ФТТ 62, 1244, (2020).

[23] A.L. Korzhenevskii, R. Bausch, R. Schmitz. Phys. Rev. E 83, 041609 (2011).

Редактор Е.Ю. Флегонтова 